\title{
Hyporesponsiveness to intradermal administration of hepatitis B vaccine in insulin dependent diabetes mellitus
}

\author{
S Li Volti, M Caruso-Nicoletti, F Biazzo, A Sciacca, G Mandarà, M Mancuso, F Mollica
}

\begin{abstract}
The immune response to intradermal or intramuscular hepatitis $B$ vaccine in 18 children with insulin dependent diabetes mellitus (IDDM) compared with 24 healthy children was studied. Patients were divided into responders, hyporesponders, and non-responders according to their antihepatitis $B$ serum concentrations after hepatitis $B$ vaccination. We also studied HLA class II antigen distribution and did delayed type hypersensitivity (DTH) tests on children with IDDM and controls.
\end{abstract}

No difference in the immune response (antihepatitis B surface antigen antibody titres) was found with intramuscular administration, whereas with intradermal administration a statistically lower immune response $(p<0.001)$ was observed in children with IDDM $v$ controls. This hyporesponsiveness cannot be attributed to HLA class II antigen distribution because their frequency was the same in both groups of children with IDDM.

It is suggested that the poor immune response to intradermal hepatitis $B$ vaccine may be due to impaired macrophage activity resulting in failure of antigen presentation, which may be of importance in the immune dysfunction in children with IDDM. This hypothesis is suggested by a significantly lower score on a DTH test to a battery of antigens in the IDDM group when compared with controls. It is therefore suggested that when the hepatitis $B$ vaccination is offered to children with IDDM it may be preferable to give it intramuscularly.

(Arch Dis Child 1998;78:54-57)

Keywords: hyporesponsiveness; hepatitis B vaccine; cell mediated immunity

Children with insulin dependent diabetes mellitus (IDDM) are at increased risk of hepatitis $\mathrm{B}$ virus (HBV) infection. ${ }^{12}$ The hepatitis $\mathrm{B}$ vaccine is therefore recommended at least in countries where $\mathrm{HBV}$ is endemic. Unfortunately, the immune response to hepatitis B vaccine is lower in patients with IDDM than in normal subjects. ${ }^{3-5}$ This is probably due to genetic factors as genes present in the major histocompatibility complex play a central part in the modulation of the immune response to hepatitis B surface antigen stimulation (HBsAg) ${ }^{67}$ The immune response to hepatitis
B vaccine is favoured by HLA-A11, which is present more frequently in responders, while HLA-DR3, DR4, DR7, and B8 have an increased prevalence among hyporesponders and non-responders as well as among patients with IDDM. $6{ }^{6}{ }^{9}$ For this reason, giving hepatitis $B$ vaccine intradermally could represent an alternative route of immunisation for these patients. Indeed, this procedure, apart from being equally safe, seems more effective and appropriate in subjects with no or poor immune response to the conventional route of giving the hepatitis $\mathrm{B}$ vaccine. ${ }^{10-12}$ Moreover, it is a useful and rapid method of enhancing the immune response. ${ }^{13}$

The aim of this study was to compare the immune response to hepatitis $\mathrm{B}$ vaccine given either intradermally or intramuscularly in children with IDDM.

Patients and methods

The children enrolled in this study were divided into two cohorts.

\section{COHORT 1}

Cohort 1 included 18 children with IDDM (11 boys and seven girls) who regularly attended the endocrine clinic of our department and were seronegative for $\mathrm{HBsAg}$ and other $\mathrm{HBV}$ markers. Their mean (SD) age was 9.1 (4.0) years and their ages ranged between 3 and 17.2 years. The mean (SD) duration of diabetes was 3.4 (2.1) years, ranging between 0.2 and 9 years. They received two to four injections of insulin per day and the mean (SD) concentration of glycated haemoglobin $\left(\mathrm{HbA}_{\mathrm{c}}\right)$ was $7.8 \%(1.5 \%)$. The individual $\mathrm{HbA} 1_{c}$ value was calculated from the mean of three to four values obtained in the previous year. None of the patients had diabetic complications. The patients were randomly divided into two numerically identical groups (A and B). Patients in group A (mean (SD) age 9.8 (4.0) years; mean (SD) duration of diabetes 3.3 (1.5) years; mean (SD) $\left.\mathrm{HbA1}_{\mathrm{c}} 9.4 \%(1 \%)\right)$ received $3 \mu \mathrm{g}$ of a recombinant DNA hepatitis B vaccine (Engerix B, Smith-Kline Beecham Biologicals, Rixensart, Belgium) intradermally in the volar face of their left forearm at the start of the study and then at two, four, and six or eight week intervals. Patients in group B (mean (SD) age 8.4 (4.0) years; mean (SD) duration of diabetes 3.4 (1.8) years; mean (SD) $\mathrm{HbA} 1_{\mathrm{c}}: 8.3 \%$ $(1.6 \%))$ were given three doses of $10-20 \mu \mathrm{g}$ (the higher dose was injected to patients older than 10 years) of the same vaccine intramuscularly in their left thigh at the start of the study 
and then after one and six months. The vaccine was not injected in the sites where insulin was usually given.

COHORT 2

Cohort two included 24 normal children (15 boys and nine girls) referred to our clinic for short stature and in whom diagnosis of familiar short stature was made. They were selected among those who had not been vaccinated so as to guarantee that age and sex distributions matched those of groups A and B. These children were also randomly divided into two numerically identical groups (A1 and B1) and received the same vaccine schedule as groups $A$ and $\mathrm{B}$. The mean (SD) age of children of group A1 was 6.3 (1.8) years and that of children of group B1 was 7.0 (1.2) years. No statistically significant difference was found either between group $\mathrm{A}$ and $\mathrm{B}$ for age, sex, duration of diabetes, or $\mathrm{HbA} 1_{c}$, or between group $\mathrm{A}$ and $\mathrm{A} 1$ and $\mathrm{B}$ and $\mathrm{B} 1$ for age and sex.

After written informed parental consent was received and before intradermal or intramuscular hepatitis B vaccine was given, a sample of venous blood $(10 \mathrm{ml})$ was taken from children with IDDM and from controls in order to evaluate: (1) serum concentrations of HBsAg, HBsAg antibodies (antihepatitis B), and other common markers of HBV infection (Ausria II, Corab and Ausab, Abbott Laboratories, North Chicago, IL, USA). None of the subjects in the four groups was seropositive; (2) HLA class I (B8) determined by serological typing for HLA-A, B, and C antigens, and HLA class II (DR3, DR4, and DR7) determined by polymerase chain reaction (PCR) (PCR by molecular typing for HLA DRB) (Amplicor, HLA DRB test, Roche); (3) serum concentrations of antibodies against diphtheria and tetanus toxoids and trivalent oral polio virus vaccine (OPV) of types 1,2 , and 3 vaccine given at ages 3, 5, and 11 months. The titres of antibodies against diphtheria and tetanus toxoids were determined by passive haemoagglutination technique. Neutralising titres for antibodies to OPV types 1, 2, and 3 were assayed in tissue culture plates containing vero cell monolayers. Titres greater than $0.01 \mathrm{IU} / \mathrm{ml}$ for diphtheria and tetanus and $1 / 4$ for OPV types 1,2 , and 3 were considered as protective.

A delayed type hypersensitivity (DTH) test was used to evaluate cell mediated immunity in IDDM and controls by a multiple intradermal antigen dispenser (Multitest IMC, Pasteur Merieux, Lyon, France). Seven different antigens (tetanus, diphtheria, streptococcus, tuberculin, candida, trichophyton, proteus) and a glycerine control were applied on the children's right forearm (through an applicator, preloaded with the tested antigens) and the induration for each antigen tested was measured 48 hours later. The induration size was carefully measured using the two diameters and the mean values recorded. Only reactions with diameters above $2 \mathrm{~mm}$ were considered positive. A DTH score reflecting the responsiveness to all antigens was then calculated for each child. The calculation was based on the total of the diameters above $2 \mathrm{~mm}$ for the seven tested antigens.

Other blood samples were obtained four to five weeks after the completion of hepatitis B vaccine administration from children with IDDM and normal children for anti-HBs evaluation. According to their anti-HBs serum concentrations, patients were considered responders ( $>100 \mathrm{mIU} / \mathrm{mml}$ ), hyporesponders $(>10<100 \mathrm{mIU} / \mathrm{ml})$, or non-responders $(<10$ $\mathrm{mIU} / \mathrm{ml}$ ). Non-responders were given an additional dose of hepatitis $\mathrm{B}$ vaccine, and anti-HBs serum concentrations were checked four to five weeks later.

Statistical analysis was performed using the $\chi^{2}$ test, the $\chi^{2}$ test for trend, and the Student's $t$ test after transformation of the data in order to compare the median and the quartiles of antiHBs in children with IDDM and controls, as well as the linear regression analysis for correlations.

\section{Results}

Table 1 shows the number of responders, hyporesponders, and non-responders in all groups. Subjects in the control groups showed effective immune response to being given hepatitis $B$ vaccine and therefore were all considered responders. Children with IDDM who were given intramuscular hepatitis B vaccine (group B) also showed effective immune responses to hepatitis $B$ vaccine, with the exception of one patient who was classified as a hyporesponder. In contrast, among children with IDDM who received intradermal hepatitis B vaccine (group A) we found two non-responders, five hyporesponders, and only two responders. The percentage of responders among children with IDDM of this group was significantly lower than in controls $(p<0.001)$ and in group $B$ children with IDDM ( $<<0.01)$.

Table 2 shows the median and the quartiles of anti-HBs (mIU/ml) in all groups of children. Significantly lower median of anti-HBs was found in group A children with IDDM when compared with controls $(\mathrm{p}<0.001)$ and with group B children with IDDM ( $p<0.001)$. No statistically significant difference was observed when this last group of children was compared with controls. Children with IDDM who were non-responders received a fourth dose of hepatitis B vaccine intradermally, but did not

Table 1 Number of responders (anti-HBs $>100$ $\mathrm{mIU} / \mathrm{ml})$, hyporesponders $(10<$ anti-HBs $<10 \mathrm{mIU} / \mathrm{ml})$ and non-responders (anti-HBs $<10 \mathrm{mIU} / \mathrm{ml})$ after the complete course of hepatitis $B$ vaccination in children with IDDM (A and B) and in controls (A1 and B1) who were given hepatitis $B$ vaccine intradermally ( $A$ and $A 1)$ or intramuscularly (B and B1)

\begin{tabular}{cclll}
\hline & No & Responders & Hyporesponders & Non-responders \\
\hline IDDM & & & 5 & 2 \\
A (id) & 9 & 2 & 1 & 0 \\
B (im) & 9 & 8 & & \\
Controls & & & 0 & 0 \\
A1 (id) & 12 & 12 & 0 & 0 \\
B1 (im) & 12 & 12 & 0 & \\
\hline
\end{tabular}

A $v$ A $1 \chi^{2}=13.99 ; \mathrm{p}<0.001$.

$\mathrm{A} v \mathrm{~B} \chi^{2}=8.26 ; \mathrm{p}<0.01$

$\chi^{2}$ for trend (two degrees of freedom) $=9.54 \mathrm{p}<0.05$.

id = intradermal; im = intramuscular. 
Table 2 Median and quartiles of anti-HBs ( $\mathrm{mIU} / \mathrm{ml})$ after the completion of the hepatitis $B$ vaccine administration in IDDM children $(A$ and $B)$ and in controls ( $A 1$ and B1) after the intradermal $(A$ and $A 1)$ or intramuscular ( $B$ and $B 1$ ) hepatitis $B$ vaccination.

\begin{tabular}{ll}
\hline & Median (interquartile range) \\
\hline IDDM & \\
A (id) $n=9$ & $27(0-135)$ \\
B (im) $n=9$ & $3000(2365-3000)$ \\
Controls & \\
A1 (id) $n=12$ & $1593(1020-3000)$ \\
B1 (im) $n=12$ & $1827(653-3000)$
\end{tabular}

Both A $v$ B and A $v$ A1 $=\mathrm{p}<0.001$ by Student's $t$ test on logarithmic data with correction for 0 values.

id = intradermal; im = intramuscular.

show any statistically significant increase in titres of anti-HBs when compared with those obtained after receiving the third dose of hepatitis $B$ vaccine.

As expected, in all children with IDDM HLA class II antigens DR3 and DR4 were more prevalent than in controls $(50 \% v 21 \%$ for DR3 and $65 \%$ v $25 \%$ for DR4). However, the immune response was not affected by HLA types (data not shown).

There was no statistically significant difference in titres of antibodies against diphtheria and tetanus toxoids and OPV virus types 1,2 , and 3 in children with IDDM (A and B) and controls (A1 and B1).

The number of positive responses $(>2 \mathrm{~mm})$ to the seven tested antigens $(1.50(0.89 \mathrm{SD}) v$ 3.85 (1.42 SD)) and the mean (SD) DTH score $(7.30 \mathrm{~mm}(5.93 \mathrm{~mm})$ v $14.92 \mathrm{~mm}(7.35$ $\mathrm{mm})$ ) were both significantly lower $(\mathrm{p}<0.001$ and $\mathrm{p}<0.01$, respectively) in children with IDDM compared with controls.

Figure 1 shows a significant correlation $(\mathrm{p}<$ 0.001 ) between titres of anti-HBs and the mean DTH score in children with IDDM who were vaccinated intradermally. No correlation was found either among titres of anti-HBs and DTH score in groups $\mathrm{B}, \mathrm{A} 1$, and $\mathrm{B} 1$ or among titres of anti-HBs and age, duration of the disease, or $\mathrm{HbAl}_{\mathrm{c}}$ in groups $\mathrm{A}$ and $\mathrm{B}$.

\section{Discussion}

Our study shows no significant difference between children with IDDM and controls in their immune responses to intramuscular hepatitis $\mathrm{B}$ vaccine. However, there was a poorer immune response to intradermal hepatitis $B$ vaccine in children with IDDM (group A). This hyporesponsiveness does not seem to be correlated with HLA II class antigen distribution as there were no differences among

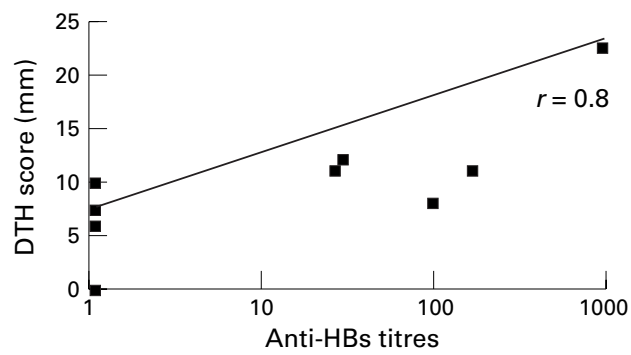

Figure 1 Correlation between the mean DTH score and the serum concentrations of anti-HBs (logarithmic data) in children with IDDM vaccinated intradermally. responders, hyporesponders, and nonresponders, nor does it appear to be due to age or duration of diabetes. Some evidence suggests that patients with IDDM show immunological disturbances in cell mediated immunity in which macrophages should play a central part in antigen presentation for antibody production by B cells. ${ }^{14-19}$ Our results, although the number of patients examined is small, support this evidence. As has been previously reported in our series, children with IDDM have a defect in cell mediated immunity as suggested by the low number of positive responses to tested antigens and low DTH score when compared with controls. ${ }^{20}{ }^{21}$ The low DTH score was not influenced by the previous exposure to the antigen used because serum concentrations of antibodies against diphtheria and tetanus toxoids and OPV virus types 1,2 , and 3 were similar in children with IDDM and in controls. Therefore in these patients there may be impaired macrophage activity causing a failure in antigen presentation. In our opinion this abnormality in the immune system may be responsible for the lower number of responders and lower median anti-HBs titre in children with IDDM receiving vaccine intradermally as compared with those receiving vaccine intramuscularly. This hypothesis is further supported by the finding that there is a significant correlation between antibody responses to hepatitis $B$ vaccine intradermally and cutaneous reactivity to Multitest. Therefore where hepatitis $\mathrm{B}$ vaccine is offered to children with IDDM, intramuscular administration may be preferable in order to guarantee adequate and long term protection against HBV infection.

1 Madalinski K, Brzosko W, Malewski M, et al. Australian antigen in sera of diabetic patients. Lancet 1971;i:701-2.

2 Polish LB, Shapiro CN, Boner F, et al. Nosocomial transmission of hepatitis B virus associated with use of a spring-loaded finger-stick device. $N$ Engl f Med 1992;326: $721-5$.

3 Pozzilli P, Arduini P, Visalli M, et al. Reduced protection against hepatitis $\mathrm{B}$ virus following vaccination in patients with type 1 (insulin-dependent) diabetes. Diabetologia 1987;30:817-9.

4 Wismans PJ, von Hattum J, de Gast GC, et al. A prospective study of in vitro anti-HBs producing B-cells (Spot-ELISA) following primary and supplementary vaccination with recombinant hepatitis B vaccine in insulin dependent diabetic patients and matched controls. $7 \mathrm{Med}$ Virol 1991;35:216-22.

5 Palla G, Calisti L, Crespin L, et al. Vaccinazione anti-epatite $B$ in un gruppo di bambini con diabete mellito insulino dipendente: valutazione della risposta anticorpale e following a 6 e 12 mesi. Riv Ital Ped Prev Soc 1994;44:176.

6 Craven DE, Awdeh ZL, Knuches LM, et al. Non responsiveness to hepatitis $B$ vaccine in health care responsiveness to hepatitis $\mathrm{B}$ vaccine in
workers. Ann Intern Med 1986;105:355-60.

7 Alper CA, Krunskall MS, Margus-Bagley D, et al. Genetic prediction of non response to hepatitis B vaccine. $N$ Engl f prediction of non resp

8 Walker M, Szmuness W, Stevens C, et al. Genetics of anti-hepatitis Bs responsiveness HLA-DR7 and nonresponsiveness to hepatitis vaccination. Proceedings of the American Association of Blood Banks 1981;5:601 (abstract 21).

9 Egea E, Iglesias A, Salazar M, et al. The cellular basis for lack of antibody response to hepatitis B vaccine in humans. f Exp Med 1991;173:531-5.

10 Miller KD, Gibbs RD, Mulligan MM, et al. Intradermal hepatitis $\mathrm{B}$ virus vaccine: immunogenicity and side-effects in adults. Lancet 1983;ii: $1454-6$.

11 Bryan JP, Sjogren MH, Perine PL, et al. Low-dose intradermal and intramuscular vaccination against hepatitis B. Acta Paediatr 1992;81:935-6.

12 Nagafuchi S, Kashiwagi S, Okada K, et al. Reversal of nonresponders and postexposure prophylaxis by intradermal hepatitis $\mathrm{B}$ vaccination in japanese medical personnel. FAMA 1991;265:2679-83. 
13 Leonardi S, Leggio T, Sciacca A, et al. Intradermal hepatitis B vaccination in thalassemia. Arch Dis Child 1990;65:527-

14 Pozzilli P, Zuccarini O, Tavicoli M, et al. Monoclonal antibodies define abnormalities of $\mathrm{T}$ lymphocyte subsets in diabetes. Diabetes 1983;32:91-4

15 Drell DW, Notkins AL. Multiple immunological abnormalities in patients with type 1 (insulin-dependent diabetes mellitus. Diabetologia 1987;30:132-43.

16 Lorini R, Montagna D, Lanfranchi A, et al. Alterations of in vitro interleukin 1 and 2 in diabetic children. Eur $\mathcal{F}$ Pediat 1989;148:732-4.

17 Paul WE. The immune system: introduction. In: Wingaarden JB, Smith LH, eds. Cecil textbook of medicine. Philadelphia: WB Saunders, 1990:1932-7.
18 Sahdev I, Fort P, Herry A. Macrophage-released interleukin- 1 in patients with type 1 diabetes mellitus. Acta Paediatr 1992;81:935-6.

19 Bellanti JA, Rocklin RE. Cell-mediated immune reactions. In: Bellanti JA, ed. Immunology III. Philadelphia: WB Saunders, 1985:176-88.

20 Pozzilli P, Pagani S, Arduini P, et al. In vivo determination of cell mediated immune response in diabetic patients using a multiple intradermal antigen dispenser. Diabetes Res 1987; 6:5-8.

21 Savagnone E. Studio multicentrico sul rischio infettivo del diabetico. Nota I. Correlazione tra alterato metabolismo glicidico e cutireattivita al Multitest IMC. Minerva Endocrinol 1991;16:119-25. 